\title{
Cerebral Aspergillus arteritis with bland infarcts: A report of two patients with poor outcome
}

\author{
Megha S. Uppin, Sundaram Challa, Shantveer G. Uppin, Suvarna Alladi*, Jyotsna Rani Yarlagadda** \\ Departments of Pathology, *Neurology, ${ }^{* *}$ Radiology and Imageology, NIMS, Panjagutta, Hyderabad, Andhra Pradesh, India
}

\begin{abstract}
Two patients with cerebrovascular aspergillosis, in the form of arteritis, thrombosis and bland infarcts are reported. One patient had systemic lupus erythematosus with disseminated aspergillosis in lungs, kidneys and brain. The other patient was immunocompetent and had sphenoid sinusitis. Both the patients were diagnosed at autopsy only, despite extensive imaging and laboratory studies. High index of clinical suspicion and early aggressive antifungal therapy are required since definite diagnostic modalities are not available.
\end{abstract}

Key words: Aspergillus, cerebral arteritis, early diagnosis infarct, systemic lupus erythematosus

\section{Introduction}

Aspergillus mycotic aneurysm though rare, is a welldescribed entity in the literature. However, cerebral arteritis due to Aspergillus, producing thrombosis and large infarcts is extremely uncommon. ${ }^{[1-4]}$ We present two patients with Aspergillus arteritis of cerebral vessels with bland infarcts.

\section{Case Reports}

\section{Case 1}

An 18 year old female presented with complaints of puffiness of face, malar rash, oral apthous ulcers and high grade fever of one month duration. On investigation, she had anti-dsDNA and anti-Sm antibodies, neutrophilic leucocytosis (12500 cells/cmm), albuminuria, hematuria and pyuria with granular casts. The chest radiograph showed patchy areas of consolidation and homogenous opacity in left lower lobe. She was diagnosed as systemic lupus erythematosus (SLE) with pneumonia and urinary tract infection and was started on broad-spectrum antibiotics.

Five days later, she developed respiratory arrest, became unconsious and was connected to ventilator. A magnetic resonance imaging (MRI) of brain showed diffuse cerebral edema and enlarged brain stem. There was hypo intensity on T1W1 and isointensity on T2W2 images. Flow void of basilar artery was lost with hyperintensities.

She deteriorated further and expired four days later.

At autopsy, basilar artery was occluded with thrombus and vessel wall was invaded by slender septate hyphae branching at acute angle, better delineated on silver methenamine stain [Figure 1]. Morphology was consistent with Aspergillus species. Cerebrospinal fluid (CSF) obtained at autopsy was sterile on culture. The brain stem, cerebellum, temporal and parietal lobes and left basal ganglia showed infarction without evidence of organisms. There was no involvement of brain by lupus. The lungs and kidneys showed hemorrhagic infarcts with Aspergillus hyphae and kidneys in addition showed class III lupus nephritis. Heart did not reveal any valvular vegetation.

\section{Case 2}

A 28-year-old male presented with high-grade fever and

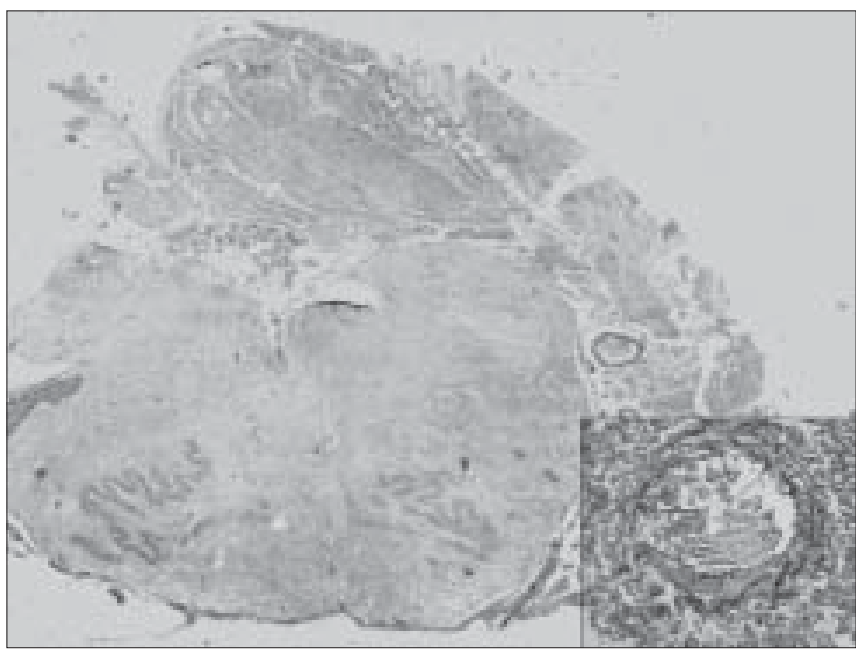

Figure 1: Whole mount section of brain stem at the level of inferior olivary nucleus showing infarction. (H \& E, x20) Inset: Basilar artery infiltration by Aspergillus hyphae (SM, x400) 
headache of 25 days duration. He was unconscious at admission and examination revealed right hemiparesis. Chest radiograph was normal. MRI of brain revealed focal hyper intensities on T2W2 and FLAIR images, in pons, bilateral thalami and left capsuloganglionic region, suggestive of acute infarcts. There was also small T2 hyper intense area in left basal ganglia partly suppressed on FLAIR suggestive of an old infarct [Figure 2a]. There was mucosal thickening of sphenoid sinus. CSF showed lymphocytic pleocytosis with elevated protein (100 mg\%) and reduced sugar (50 mg\%). Smear and culture were negative for bacteria, fungi and acid-fast bacilli. The 2D echo was normal. There was neutrophilic leucocytosis (10800 cells/cmm). Patient was diagnosed as chronic meningitis with vasculitis and started on antibiotics and steroids.

On fifth day patient developed decerebrate posturing and was connected to ventilator. Computed tomography (CT scan) revealed hypodensity with mass effect suggestive of acute to sub-acute global infarct involving entire left cerebral hemisphere [Figure 2b].

His condition worsened further and he expired on seventh day of admission. At autopsy, brain was markedly edematous with basal exudates. There was thickening and narrowing of lumen of left internal carotid artery (ICA), left middle cerebral artery (MCA) and basilar artery (BA) [Figure 3]. Coronal sections revealed softening of the entire left cerebral hemisphere, brainstem and cerebellum.

Sections from ICA, MCA and BA showed occlusion of the lumen by an organizing thrombus with multiple septate, acute angle branching hyphae of Aspergillus species invading the vessel wall and extending into the lumen.

There were no valvular vegetations and the lung showed bronchopneumonia. The sphenoid mucosa sampled at autopsy showed dense neutrophilic infiltrate. However, fungal stains were negative.
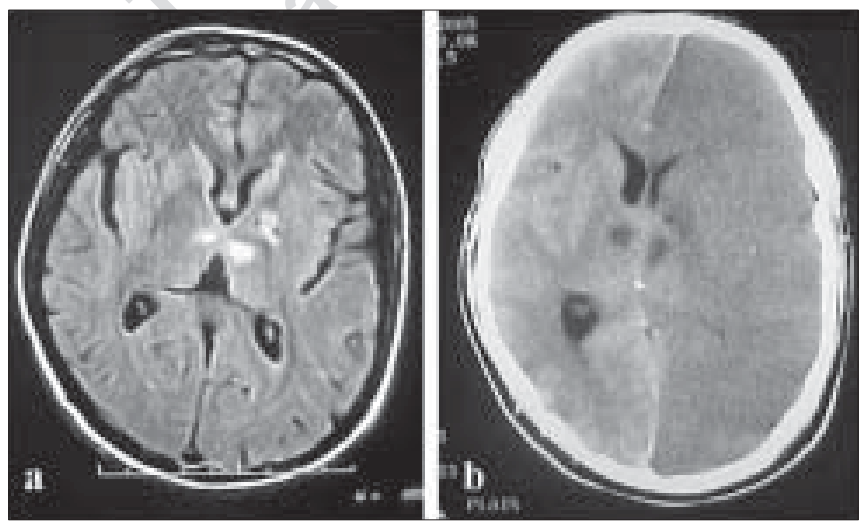

Figure 2: (a) T2W and FLAIR MR Image showing focal hyperintensities in bilateral thalami and left capsuloganglionic region (b) CT image showing global infarct of the left cerebral hemisphere corresponding to left middle cerebral artery territory

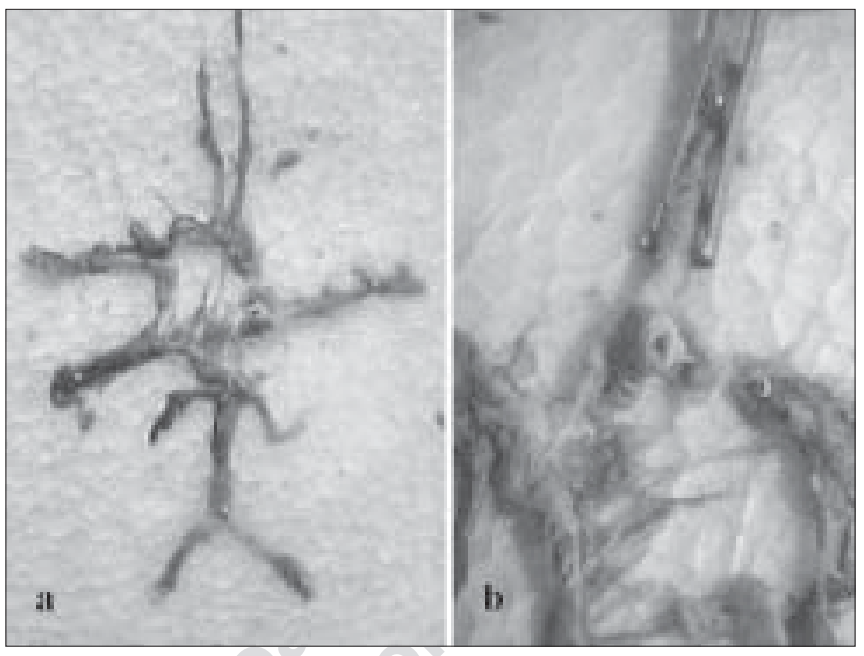

Figure 3: (a) Circle of Willis showing thickened left internal carotid artery and middle cerebral artery with narrowed lumina. (b) closer view of A

\section{Discussion}

Central nervous system aspergillosis results due to spread from contiguous structures like paranasal sinus orbit, skull bone or ear or hematogenous spread from lung, heart or gastrointestinal tract. It can occur in both immunosuppressed and immunocompetent individuals. Invasive aspergillosis, particularly sinocranial aspergillosis in apparently immunocompetent persons is reported from India. ${ }^{[5-7]}$

Lung is the source of infection in disseminated disease in immunosuppressed individuals as seen in our patient with SLE and route of spread is hematogenous. Isolated involvement of any paranasal sinus, particularly sphenoid sinus by aspergillosis is rare. ${ }^{[5]}$ The central location of sphenoid sinus in the skull base provides multiple pathways for intracranial spread of infection to critical regions of CNS and cerebral vasculature. ${ }^{[8,9]}$ Arteritis of major vessels with thrombosis and infarction as seen in our patients is extremely uncommon. The source of Aspergillus infection was uncertain in our case 2 who had no fungal hyphae in the sinus mucosa or evidence of valvular vegetations.

Cerebrovascular aspergillosis occurs from endovascular infection from a septic embolus or vasculitis. ${ }^{[10]}$ Virulent organisms produce arteritis and less virulent organisms produce mycotic aneurysms. ${ }^{[11]}$ The angioinvasive nature of Aspergillus is directly related to its ability to digest elastic tissue by production of the enzyme elastase. The enzyme elastase digests the barriers to the spread of infection in the tissues including the elastin in the arterial walls. Animal studies and human studies showed higher mortality and aggressive clinical course with strains of Aspergillus that produce elastase compared to the ones that do not. ${ }^{[12,13]}$ Vessel invasion and extension of hyphae into lumen causes thrombosis 
and embolisation of hyphal masses. The tendency to invade proximal vessels results in infarcts affecting large vascular distributions as seen in both our patients.

The diagnosis of CNS aspergillosis remains a challenge. The clinical manifestations are protean and non-specific. Imaging studies do not help in etiologic diagnosis. In both our patients, diagnosis was not made antemortem despite extensive investigation and both were treated for possibility of bacterial infections.

Laboratory diagnosis of aspergillosis also poses problems, as blood and CSF cultures are invariably negative as seen in both our patients. Tissue biopsy is almost always necessary for diagnosis. However, in pathologies like arteritis, tissue biopsy is inappropriate. CSF may show pleocytosis as seen in our patient and does not contribute to etiologic diagnosis.

As early diagnosis is essential for treatment and classic microbiological procedures have limitations, several non-culture-based diagnostic methods for invasive aspergillosis are being developed which include whole blood polymerase chain reaction (PCR), galactomannan (a major cell wall protein of Aspergillus) sandwich enzyme linked immunosorbant assay (ELISA), Western blot to detect antibodies and fungal metabolites like D-arabinitol and 1,3-beta-D glucan. ${ }^{[14,15]}$ A PCR test in combination with galactomannan antigen testing is useful in diagnosing invasive aspergillosis. ${ }^{[16]}$

The rarity of the lesion, rapid progression to poor outcome and difficulty in diagnosis warrant a high index of clinical suspicion. Cerebral vascular involvement, on imaging in sinusitis or immuno-suppressed patient should warrant serological methods for establishing diagnosis of fungal etiology. Early and aggressive antifungal therapy with amphotericin B should be instituted. New antifungal agents such as voriconazole, posaconazole, caspofungin may have a role to play. However, due to rarity of antemortem diagnosis of Aspergillus arteritis, the efficacy of any of these drugs is not known.

\section{References}

1. McKee EE. Mycotic infection of the brain with arteritis and sub-arachanoid hemorrhage: Report of case. Am J Clin Pathol 1950;20:381-4.

2. Endo T, Tominaga T, Konno H, Yoshimoto T. Fatal subarachnoid hemorrhage, with brainstem and cerebellar infarction, caused by Aspergillus infection after cerebral aneurysm surgery: Case report. Neurosurgery 2002;50:1147-51.

3. Corvisier N, Gray F, Gherardi R, Lebras F, Blanc CM, Nguyen JP, et al. Aspergillosis of ethmoid sinus and optic nerve, with arteritis and rupture of the internal carotid artery. Surg Neurol 1987;28:311-5.

4. Lau AH, Takeshita M, Ishii N. Mycotic (Aspergillus) arteritis resulting in fatal subarachnoid hemorrhage: A case report. Angiology 1991;42:2515 .

5. Chopra H, Dua K, Malhotra V, Gupta RP, Puri H. Invasive fungal sinusitis of isolated sphenoid sinus in immunocompetent subjects. Mycoses 2006;49:30-6.

6. Murthy JM, Sundaram C, Prasad VS, Purohit AK, Rammurti S, Laxmi V. Sinocranial aspergillosis: A form of central nervous system aspergillosis in South India. Myeoses 2001;44:141-5.

7. Sundaram C, Umabala P, Laxmi V, Purohit AK, Prasad VS, Panigrahi M, et al. Pathology of fungal infections of the central nervous system: 17 years' experience from Southern India. Histopathology 2006;49:396405 .

8. Bazan C, Rinaldi M, Rauch R, Jinkins J. Fungal infections of the brain. Neuroradiol Clin N Am 1991;1:57-88.

9. Kountakis SE, Kemper JV Jr, Chang CY, DiMaio DJ, Stiernberg CM. Osteomyelitis of the base of the skull secondary to Aspergillus. Am J Otolaryngol 1997;18:19-22.

10. Boes B, Bashir R, Boes C, Hahn F, McConnell JR, McComb R. Central nervous system aspergillosis. Analysis of 26 patients. J Neuroimaging 1994;4:123-9.

11. Davidson P, Robertson DM. A true mycotic (Aspergillus) aneurysm leading to fatal subarachnoid hemorrhage in a patient with hereditary hemorrhagic telangiectasia. Case report. J Neurosurg 1971;35:71-6.

12. Kothary MH, Chase T Jr, MacMillan JD. Correlation of elastase production by some strains of Aspergillus fumigatus with ability to cause pulmonary invasive aspergillosis in mice. Infect Immun 1984;43:3205.

13. Rhodes JC, Bode RB, McCuan-Kirsch CM. Elastase production in clinical isolates of Aspergillus. Diagn Microbiol Infect Dis 1988;10:165-70.

14. Verweij PE, Brinkman K, Kremer HP, Kullberg BJ, Meis JF. Aspergillus meningitis: Diagnosis by nonculture based microbiological methods and management. J Clin Microbiol 1999;37:1186-9.

15. Reiss E, Obayashi T orle K, Yoshida M, Zancope-Oliveira RM. Nonculture based diagnostic tests for Mycotic infections. Med Mycol 2000;38:147-59.

16. White PL, Linton CJ, Perry MD, Johnson EM, Barnes RA. The evolution and evaluation of a whole blood polymerase chain reaction assay for the detection of invasive aspergillosis in hematology patients in a routine clinical setting. Clin Infect Dis 2006;42:479-86.

Accepted on 15-09-2006

Source of Support: Nil, Conflict of Interest: None declared. 